\title{
Fluid-structure interactions enable passive flow control in real and biomimetic plants
}

Park, Keunhwan; Tixier, Aude; Paludan, Magnus; Østergaard, Emil; Zwieniecki, Maciej; Jensen, Kaare H.

Published in:

Physical Review Fluids

Link to article, DOI:

10.1103/PhysRevFluids.6.123102

Publication date:

2021

Document Version

Publisher's PDF, also known as Version of record

Link back to DTU Orbit

Citation (APA):

Park, K., Tixier, A., Paludan, M., Østergaard, E., Zwieniecki, M., \& Jensen, K. H. (2021). Fluid-structure interactions enable passive flow control in real and biomimetic plants. Physical Review Fluids, 6(12), [123102]. https://doi.org/10.1103/PhysRevFluids.6.123102

\section{General rights}

Copyright and moral rights for the publications made accessible in the public portal are retained by the authors and/or other copyright owners and it is a condition of accessing publications that users recognise and abide by the legal requirements associated with these rights.

- Users may download and print one copy of any publication from the public portal for the purpose of private study or research.

- You may not further distribute the material or use it for any profit-making activity or commercial gain

- You may freely distribute the URL identifying the publication in the public portal 


\title{
Fluid-structure interactions enable passive flow control in real and biomimetic plants
}

\author{
Keunhwan Park $\odot,{ }^{1, *}$ Aude Tixier $\odot,{ }^{2, \dagger}$ Magnus Paludan, ${ }^{1}$ Emil Østergaard $\odot,{ }^{1}$ \\ Maciej Zwieniecki, ${ }^{2,}$ and Kaare H. Jensen $\odot^{1, \S}$ \\ ${ }^{1}$ Department of Physics, Technical University of Denmark, Kongens Lyngby DK-2800, Denmark \\ ${ }^{2}$ Department of Plant Sciences, University of California at Davis, Davis, California 95616, USA
}

(Received 9 November 2020; revised 24 March 2021; accepted 3 June 2021;

published 13 December 2021)

\begin{abstract}
Controlling fluid flow is a fundamental problem with applications from biomedicine to environmental engineering. Contemporary solutions combine electromechanical sensors, valves, and pumps; however, these are expensive and difficult to maintain. We report an autonomous flow control principle inspired by vascular transport in plants. Combining experiments on real and biomimetic tissues, we show that networks of cells linked by nonlinear valves permit the physical programming of a nearly arbitrary pressure drop versus flow rate relation. The nonlinearity is a consequence of fluid-structure interactions that allow a flexible element to selectively block the valve aperture. We report four applications: parallel connections that function as (i) a nonlinear flow controller, (ii) a constant flow controller, (iii) a reverse Ohm flow controller, and a serial connection that acts as (iv) a fluidic on-off switch.
\end{abstract}

DOI: 10.1103/PhysRevFluids.6.123102

\section{INTRODUCTION}

Fluid flow control is a key enabling technology, with applications ranging from water mains to chemical processing, drug delivery, and robotics [1]. Present technologies combine sensors with computerized valves and pumps that accurately modify the conductivity of feed pipes or input pressure in real time. New and vital areas that require flow control at unprecedented scales are becoming evident, such as disease detection, drug infusion systems, and synthetic organs [2-4]. Other applications, in some cases arising as a consequence of climate change, such as irrigation and flood protection, are equally challenging [5,6]. However, expanding current automated technology to meet demands in all these areas is prohibitively energy intensive and expensive. This provides strong impetus for developing new, versatile, and inexpensive autonomous flow control systems.

To overcome this obstacle, we report a bio-inspired flow control concept that draws inspiration from the physics of water transport in trees. Despite the absence of cerebral matter and centralized control, plants are able to effortlessly guide water transport across networks of xylem cells that dwarf the vascular systems of all other living organisms [Fig. 1(a)]. We report here a striking feature of the xylem: when exposed to substantial pressure gradients, the xylem tissue acts as a passive constant flow controller. We exploit this feature to design programmable biomimetic plants in which almost any pressure-flow curve imaginable can be obtained.

\footnotetext{
*Present address: Department of Mechanical Engineering, Gachon University, Sujeong-gu, South Korea.

†Present address: INRAE, University of Bourgogne, Dijon, France.

‘mzwienie@ucdavis.edu

§khjensen@fysik.dtu.dk
} 

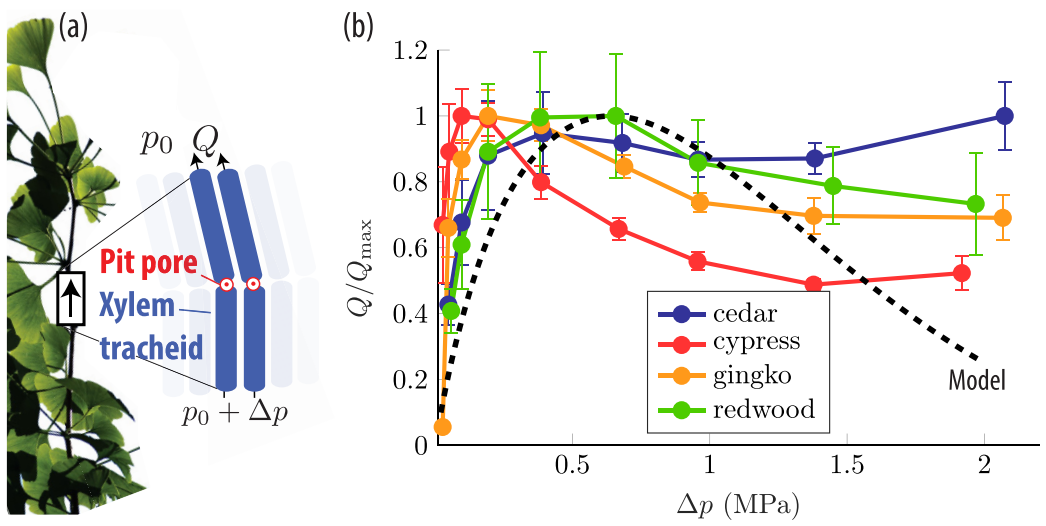

(c)

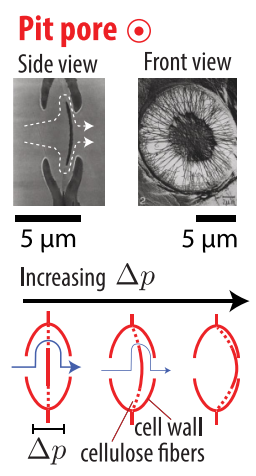

FIG. 1. Fluid-structure interactions enable passive flow control in plants. (a) Xylem cells linked by pit pores form a microfluidic network in plants to replace water lost from leaves during transpiration. (b) Normalized xylem flow rate $Q$ as a function of pressure drop $\Delta p$ across short branch segments. We observe constant flow above $\Delta p \approx 0.1 \mathrm{MPa}$. The dashed line represents the behavior of a random network model [Eq. (6), see details in the text]. (c) Microstructure of cell-to-cell torus-margo pit pores. Cellulose fibrils deform under mechanical stress, thus gradually blocking flow across the pore as the pressure reaches the critical value $\sigma$. Panel (c) is adapted from Bauch et al. [9], reproduced with permission.

Constant-flow characteristics in trees could be a consequence of the evolved resistance to embolism, a feature that prevents catastrophic loss of transport capacity due to severe drought stress $[7,8]$. It could also represent an adaptation to preventing water loss due to a sudden increase in evaporation rate on timescales faster than the stomata response time due to sun flex, hot dry wind, or tissue damage. We find that these remarkable flow features can be attributed to transport across pressure-sensitive torus-margo pit pores that link adjacent conduits in conifers. These valves contain a flexible element which can deflect to selectively block a microfluidic channel, depending on the applied pressure. Each pit pore thus acts as a pressure-sensitive nonlinear valve.

Inspired by the vegetal flow patterns, we show that simple networks of biomimetic valves can achieve nearly arbitrary pressure-drop versus flow-rate characteristics. Basic microfabrication and three-dimensional (3D)-printing techniques allow us to control the properties of the valves with integrated soft elements. Our devices are programmable and able to demonstrate user-defined target pressure-flow characteristics. We engineered different devices to demonstrate a variety of responses, including a nonlinear flow controller, a constant flow controller, an inverse Ohm flow controller, and a fluidic on-off switch.

\section{RESULTS}

More water passes through plants as part of the global water cycle than any other class of living organisms. For this reason, it is interesting to understand the basic physics of how water moves in the xylem and, in particular, how the tissue permeability depends on the physicochemical conditions. To quantify the effects of mechanical stress on the transport capacity of xylem, we conducted pressuredriven flow experiments on short branch segments. These were subjected to an axial pressure drop $\Delta p$ and we quantified the resultant liquid flow rate $Q$ [Fig. 1(b)]. (See Supplemental Material [10] for a description of the experimental methods.) Under standard physiological conditions, the cell-tocell pressure drop $\Delta p \ll 0.1 \mathrm{MPa}$, while extreme water stress corresponds to $\Delta p \approx 1 \mathrm{MPa}[11,12]$, especially in the events associated with cavitation. Across this pressure range, our experiments reveal a strikingly nonlinear pressure-flow response in cedar, cypress, gingko, and redwood, all either conifers or members of related plant families [Fig. 1(b)]. In these species, the flow rate initially increases with pressure, then peaks and saturates at an approximately constant level. In other words, 
(a)

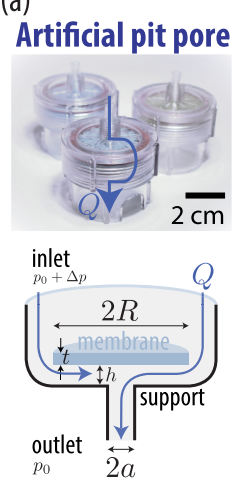

(b)

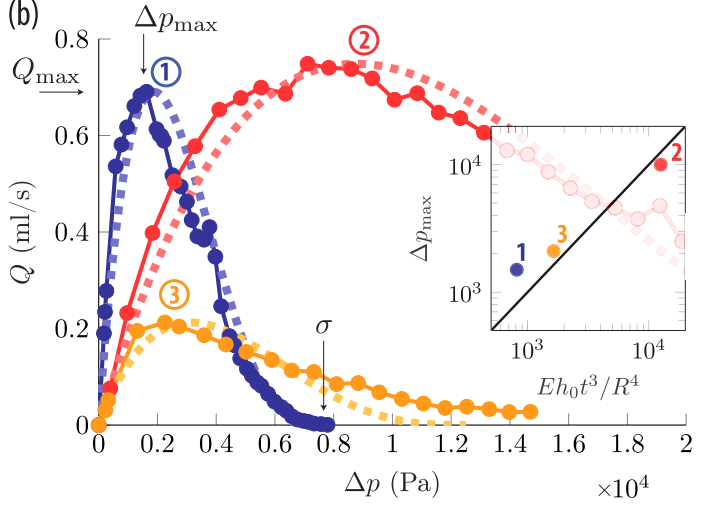

(c)
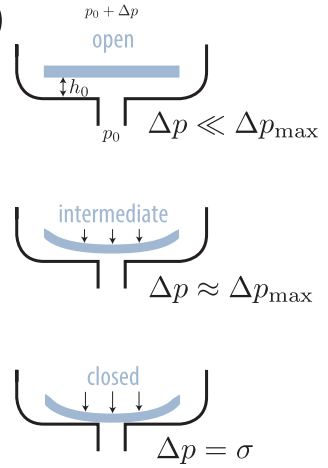

FIG. 2. Fluid-structure interactions enable passive flow control in artificial pit pores. (a) 3D-printed synthetic devices that replicate the torus-margo functionality [Fig. 1(c)]. In each device, liquid flows at a rate $Q$ past a flexible membrane which can deflect in response to the application of a pressure drop $\Delta p$ across the device. (b) Flow rate $Q$ plotted as a function of applied pressure $\Delta p$ for valves no. 1-3 (see Supplemental Material [10]). The artificial pit pores have strongly nonlinear pressure-flow characteristics and we can independently control the closing pressure $\sigma$ and maximum flow rate $Q_{\max }$ by varying the device geometry. Dashed lines indicate the analytical expression in Eq. (6). The inset shows the peak pressure $\Delta p_{\max }$ as a function of the system, in rough accord with Eq. (4). (c) Flow is restricted as the valve transitions from the open state to the intermediate and, finally, closed configuration in response to pressure.

under high pressure differences, the xylem acts as an approximately constant flow controller. This strongly contrasts with the widely presumed linear relation between pressure and flow under natural (low-pressure difference) conditions, in accord with Ohms and Darcys laws [13], which we observe when $\Delta p$ is relatively small. The underlying physical effects responsible for the observed nonlinear xylem flow control are unknown. We nevertheless note that soft materials are present in the gate structures [Fig. 1(c)]. Indeed, a particular characteristic of species that exhibit the flow in Fig. 1(b) is that the cell-to-cell torus-margo pit pores [Fig. 1(c)] contain flexible elements that could deform and impact flow [14,15].

To elucidate the underlying processes responsible for xylem flow control, we designed, fabricated, and characterized synthetic torus-margo pit pores (Fig. 2 and Supplemental Material [10]). The devices are geometrically similar to, and contain the same major components as, their biological counterparts: a membrane which can deflect to selectively block a fluidic channel, depending on the applied pressure. The synthetic pit is, however, somewhat larger than the natural system (gap height $h \approx 100 \mu \mathrm{m}$ and membrane diameter $2 R \approx 5 \mathrm{~mm}$ vs $h \approx 1 \mu \mathrm{m}$ and $2 R \approx 10 \mu \mathrm{m}$ in natural pits). Also, the flexible elements are softer (Young's modulus $E=0.9 \mathrm{MPa}$ vs $E \approx 0.5 \mathrm{GPa}$ in pits [16]). The flow speed is reasonably slow in both the plant $\left(v \approx 10^{-3} \mathrm{~m} / \mathrm{s}\right)$ and the artificial pore $\left(v \approx 10^{-1} \mathrm{~m} / \mathrm{s}\right)$. The typical Reynolds number $\operatorname{Re}=\rho v h / \eta$ is $\operatorname{Re} \approx 10^{-1}$ in the plant and $\operatorname{Re} \approx 10$ in the biomimetic system; hence the flow is dominated by viscous effects in both cases. Here, $\rho$ is the liquid density and $\eta$ is the viscosity. We note, however, that inertial effects generally lead to an increase in resistance when $\operatorname{Re}>10$ [17].

Returning to the flow characteristics of the synthetic pit pores, we observe that the flow rate $Q$ initially increases linearly with applied pressure $\Delta p$ [Fig. 2(b)]. However, after reaching a peak flow $Q_{\max }$ at the applied pressure $\Delta p_{\max }$, the current is gradually reduced. Finally, the soft valve shuts off completely at the closing pressure $\Delta p=\sigma$, which is typically 3-5 times larger than the peak pressure $\Delta p_{\max }$.

The similarity between the tissue-scale flows in xylem (Fig. 1) and the synthetic pores (Fig. 2) is notable and leads us to hypothesize that the constant flow behavior emerges from flow across a network of conduits, characteristic of wood [18]. To comprehend the performance of a network, 
however, we must first develop a basic understanding of the individual components. The flow-rate versus pressure-drop relationship for a single valve is determined by fluid-structure interactions, that is, the nonlinearity in the valves is a consequence of the elastic properties and deformability of the membrane [Fig. 2(c)]. Specifically, the applied pressure causes the membrane to deflect downwards, which in turn reduces the gap height. This leads to an increase in the resistance to flow. Because the Reynolds number is relatively small, it is reasonable to assume that the system is not too far removed from ideal Stokes flow conditions. Moreover, because the gap height $h$ is small relative to both the radius $R$ and thickness $t$ of the membrane, we can use lubrication and plate theory to explore the basic physics that control the relationship between pressure drop, deformation, and flow rate.

The pressure drop $\Delta p$ across the gap can be expressed in terms of the flow rate $Q$ and height $h$ as

$$
\Delta p=-\int_{a}^{R} \partial_{r} p d r=\frac{6 \eta Q}{\pi} \int_{a}^{R} \frac{1}{r h^{3}} d r .
$$

The height $h$ of the gap below the membrane is the crucial component in the relation between pressure drop $\Delta p$ and flow rate $Q$ in Eq. (1). When the applied pressure is relatively small, the membrane is hardly deformed and the gap height $h=h_{0}$ is constant. The pressure drop versus flow rate relation in this limit is

$$
Q=\frac{\pi}{6 \eta} \frac{h_{0}^{3}}{\ln (R / a)} \Delta p=C \Delta p,
$$

where

$$
C=\frac{\pi}{6 \eta} \frac{h_{0}^{3}}{\ln (R / a)}
$$

is the hydraulic conductance of the undeformed system. As pressure increases, however, the membrane bends down and the gap height decreases. Using linear thin-plate theory [19], we can, to a first approximation, express the gap height $h$ as

$$
h=h_{0}(1-\Delta p / \sigma), \quad \text { where } \sigma \approx E \frac{h_{0} t^{3}}{R^{4}}
$$

is the pressure required to close the valve. Here, $E$ is Young's modulus of the membrane and $t$ is its thickness. Using this gap height in Eq. (1) leads to a nonlinear pressure drop versus flow rate relation,

$$
Q \approx C \Delta p(1-\Delta p / \sigma)^{3}
$$

in reasonable accord with experiments [Fig. 2(b)]. We note that the maximum flow rate $Q_{\max }=$ $3 C \Delta p_{\max } / 4$ occurs at the peak pressure $\Delta p_{\max }=\sigma / 4$, in accord with the data (Fig. 2). The state of the pore, in both the natural and synthetic systems, is captured by the ratio $\Delta p / \sigma$ of the applied pressure $\Delta p$ to the closing pressure $\sigma$. Under standard conditions, $\Delta p / \sigma \ll 1$ and flow proceeds unhindered. However, in cases when $\Delta p / \sigma \approx 1$, flow is strongly restricted.

\section{Programmable flow in networks of nonlinear valves}

To rationalize the experimental data [Fig. 1(b)]—and potentially use the synthetic torus-margo (Fig. 2) in the design of autonomous flow control systems-we now proceed and consider pressuredriven fluid transport across a network of nonlinear flow valves [Fig. 3(a)]. Our goal is to understand the emergence of the constant-flow characteristics present in the plant data [Fig. 1(b)] and to elucidate the general flow design principles. Specifically, we inquire under what conditions it is possible to obtain an arbitrary target pressure-drop versus flow-rate relationship $Q_{t}(\Delta p)$, such as a constant flow controller, from an assembly of nonlinear valves. 
(a)
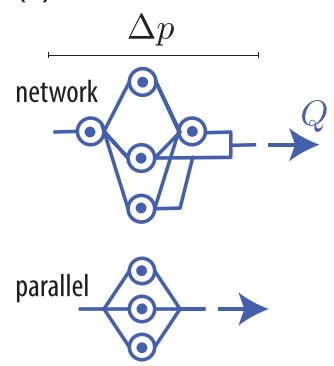

serial

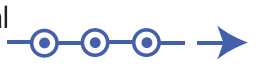

(b)

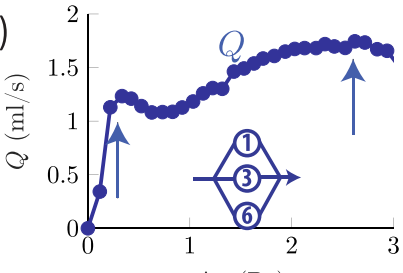

(d)

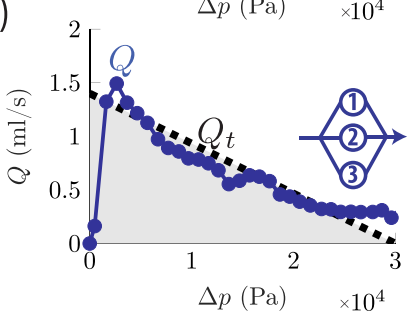

(c)

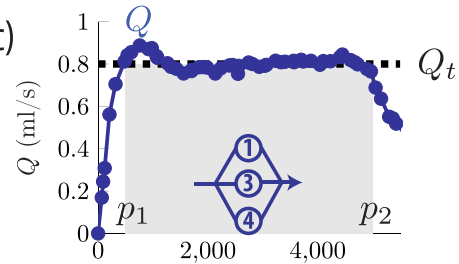

(e)

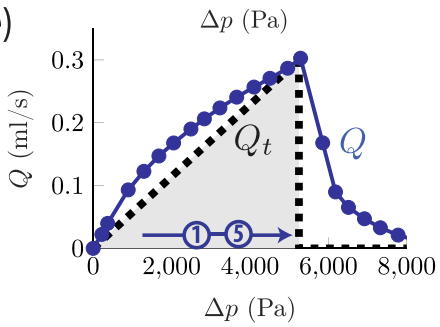

FIG. 3. Flow across assemblies of artificial pit pores. (a) Networks of nonlinear valves, such as parallel or serial couplings, allow for a wide range of flow patterns. Each circle represents a device with representative characteristics illustrated in Fig. 2(b). Straight lines symbolize rigid tubing. (b) When combining valves in parallel, we typically obtain bimodal flow characteristics (arrows). (c) By coupling specific valves in parallel, we can obtain a desired target flow rate $Q_{t}(\Delta p)$ (dashed line) across the pressure interval from $p_{1}$ to $p_{2}$ (shaded gray region), in this case constant flow $\left(Q_{t}=\right.$ const). (d) The same principle illustrated on a reverse Ohm flow, i.e., $Q_{t}=Q_{0}-k \Delta p$, corresponding to an approximately constant negative hydraulic resistance. (e) A serial coupling of two valves allows us to generate a fluidic switch. See details in the text. The individual valve properties are listed in the Supplemental Material [10].

Fluid flow in a network follows Kirchoffs laws, dictating that the algebraic sum of currents across conductors meeting at a point is zero $\left(\sum Q_{i}=0\right)$ and that the directed sum of the pressure differences around any closed loop is zero $\left(\sum \Delta p_{j}=0\right)$. Closed-form solutions for circuits are available; for a review, see, e.g., [20]. However, standard methods fail to capture flows across networks of nonlinear resistors, such as torus-margo pit pores. To unveil the basic network dynamics, it is useful first to consider the interaction of two valves, labeled $a$ and $b$, with flow rates $Q_{a}=$ $C_{a}\left(1-\Delta p_{a} / \sigma_{a}\right)^{3}$ and $Q_{b}=C_{b}\left(1-\Delta p_{b} / \sigma_{b}\right)^{3}$; cf. Eq. (5). For a parallel coupling, the same pressure drop $\Delta p=\Delta p_{a}=\Delta p_{b}$ is applied across both elements and the total flow rate $Q$ is found by adding the individual contributions, i.e., $Q=Q_{a}+Q_{b}=C_{a}\left(1-\Delta p / \sigma_{a}\right)^{3}+C_{b}\left(1-\Delta p / \sigma_{b}\right)^{3}$. This principle is illustrated in Fig. S3(a) of the Supplemental Material [10]. We will return to a more detailed discussion of parallel couplings below. Before proceeding, however, we briefly consider a serial coupling of the same elements. Here, the currents across all components, $Q=Q_{1}=Q_{2}$, are equal, but the pressure drop $\Delta p$ must be divided between the two resistors, i.e., $\Delta p=\Delta p_{a}+\Delta p_{b}$. A general feature of the $\Delta p-Q$ relation for each valve [Eq. (5)] is that there are two compatible pressures $\Delta p^{\{l, r\}}(Q)$ available for a given flow rate $Q$. Here, the superscripts $l$ and $r$ indicate values to the left or right of the peak flow rate $Q_{\max }$, respectively. For flow rates $Q>Q_{\max }$, there is no solution. The flow rate across both elements $Q$ must therefore fall in the range $0 \leqslant Q \leqslant \min \left(Q_{\max , a}, Q_{\max , b}\right)$ limited by the smallest of the two peak flow rates. For a $Q$ in this range, the two valves yield four combinations, i.e., $\Delta p=\left\{\Delta p_{a}^{l}+\Delta p_{b}^{l}, \Delta p_{a}^{l}+\Delta p_{b}^{r}, \Delta p_{a}^{r}+\Delta p_{b}^{l}, \Delta p_{a}^{r}+\Delta p_{b}^{r}\right\}$. Finally, if the pressure drop $\Delta p>\min \left(\sigma_{a}, \sigma_{b}\right)$ is greater than the smallest closing pressure $\min \left(\sigma_{a}, \sigma_{b}\right)$, there is always the option that one of the valves is closed, i.e., $Q=0$. The behavior of a basic serial coupling is illustrated in Fig. S3(b) of the Supplemental Material [10].

Our initial analysis of two network elements revealed the possibility of nonlinear pressure-flow relationships (see Fig. S3 of the Supplemental Material [10]). To expand upon these results, we proceed to consider the general case of $N$ parallel valves where the total current $Q$ corresponds to 
the sum over the individual elements,

$$
Q(\Delta p)=\sum_{n=1}^{N} C_{n} \Delta p\left(1-\frac{\Delta p}{\sigma_{n}}\right)^{3}=\sum_{n=1}^{N} C_{n} q_{n},
$$

where the index $n$ labels the characteristics of each valve and $q_{n}=\Delta p\left(1-\Delta p / \sigma_{n}\right)^{3}$. Consistent with Eq. (6), we typically observe bimodal pressure-drop versus flow-rate characteristics when linking multiple valves [Fig. 3(b)]. Experiments also confirm that the total flow is equal to the algebraic sum over the constituent valve components; see Fig. S4 of the Supplemental Material [10].

Applying the parallel coupling model in Eq. (6) to the biological data in Fig. 1(b) is not straightforward, since we do not know the precise physical properties (i.e., conductance $C$ and closing pressure $\sigma$ ) of the individual torus-margo pit pores. Also, substantial differences in vascular network architecture between species has been reported [18,21], and it is possible that some of the variation in the $Q-\Delta p$ characteristics can be attributed to structural differences. Here, we assume for simplicity that the network can be represented by a parallel coupling according to Eq. (6) and that the uncorrelated parameters are drawn from a uniform distribution. This minimal model leads to the dashed black curve in Fig. 1(b), which is not inconsistent with data, except for pressures above $\Delta p=1.5 \mathrm{MPa}$, where a clear deviation is observed, possibly due to leakage through the central disk, or via small imperfections in the seal between the disk and the cell wall. The mean closing pressure $\langle\sigma\rangle$ is the only fitting parameter, which determines the pressure at which the maximum flow occurs, $\Delta p_{\max } \approx 0.3\langle\sigma\rangle$. (The value $\langle\sigma\rangle=2 \mathrm{MPa}$ was used.) It is possible to obtain an even better fit by optimizing the probability density functions, but we will not do that here.

Having established the validity of Eq. (6) for flow across simple valve networks, and having justified that it can be used on larger sets as well, we return once again to the design question posed earlier: under what conditions is it possible to obtain an arbitrary target pressure-drop versus flow-rate relationship $Q_{t}(\Delta p)$ from an assembly of nonlinear valves? Because of the strikingly nonlinear properties of the individual valves (Fig. 2), we can conceptually treat the expression for the total flow rate emerging from a parallel coupling [Eq. (6)] as analogous to a nonorthogonal series expansion [22] of the flow rate $Q$. The basis functions are the pressure-flow curves for individual valves [Eq. (5)]. Given a desired target flow-rate function $Q_{t}$ and fixed number of valves $N$, it is in fact straightforward to determine the parameters (conductance $C_{n}$ and closing pressure $\sigma_{n}$ ) which minimize the difference between $Q$ and $Q_{t}$, either by linear algebra or by a numerical scheme (e.g., simulated annealing). To illustrate the algebraic procedure, we first consider a set of $N$ valves with known closing pressures $\sigma_{n=1, \ldots, N}$. Our goal is to determine the optimal choice of amplitudes $C_{n}$ such that the target $Q_{t}$ and actual flow $Q$ differ by as little as possible. This corresponds to minimizing the mean square difference,

$$
I=\int_{p_{1}}^{p_{2}}\left(Q_{t}-\sum_{n=1}^{N} C_{n} q_{n}\right)^{2} d(\Delta p)
$$

where $p_{1}$ and $p_{2}$ delimits the pressure interval of interest [see gray regions in Figs. 3(c)-3(e)]. Following the standard procedure used in, e.g., a Fourier series, we take the derivative with respect to $C_{m}$ in Eq. (7). This leads to a linear equation system for the valve parameters $C_{n}$, which can be readily solved using linear algebra,

$$
\sum_{n=1}^{N} C_{n} A_{n m}-B_{m}=0 \text { for } m=1,2, \ldots, N,
$$

where the matrix elements are

$$
A_{n m}=\int_{p_{1}}^{p_{2}} q_{m} q_{n} d(\Delta p) \text { and } B_{m}=\int_{p_{1}}^{p_{2}} q_{m} Q_{t} d(\Delta p) .
$$


Minimizing Eq. (7) using a fixed set of valve parameters, $\sigma_{n(n=1, \ldots, N)}$, that are, for instance, equally spaced provides a relatively close approximation to many target functions. We note, however, that in some instances the algorithm can yield unphysical negative $C_{n}$ values. To mitigate the limitations to the analytical approach, we used simulated annealing, allowing both $C_{n}>0$ and $\sigma_{n}>0$ to vary in the optimization process. For a fixed number of valves $N$, the two methods provided similar values of the optimization measure $I$ in the majority of cases.

Having established a protocol for physically programming a given pressure-drop versus flow-rate relation into geometric and material parameters, we now proceed to briefly discuss three examples (Fig. 3). With just three components, we fabricated a constant flow controller $\left[Q_{t}=\right.$ const; see Fig. 3(c)], with characteristics that are remarkably similar to plant flow data [Fig. 1(b)]. Moreover, we can program trees with many other notable flow profiles. A particularly challenging example is the reverse Ohm controller, with target flow $Q_{t}=Q_{0}-C \Delta p$, corresponding to a negative constant hydraulic resistance. In this system, flow decreases linearly with pressure. Our synthetic tree is able to achieve this with just $N=3$ components [Fig. 3(d)].

Our results illustrate that a suitable parallel superposition of valves can generate many flow characteristics. A notable exception, however, are switches that enable a rapid on-off transition in the flow characteristics. This feature, however, can exist in a serial coupling [Fig. 3(e)]. The physical mechanism responsible for the relatively rapid transition is that a given pressure drop $\Delta p$ must be distributed across the components as a sum, $\Delta p=\Delta p_{1}(Q)+\Delta p_{2}(Q)+\cdots+\Delta p_{N}(Q)$. As previously discussed, however, there are cases in which the only solution to this equation corresponds to the closed state $Q=0$, and hence the rapid transition (see also Fig. S4 of the Supplemental Material [10]).

\section{DISCUSSION AND CONCLUSION}

We have demonstrated a physical process that takes advantage of fluid-structure interactions to control fluid flows. Our results also offer insights into the physics of water transport in the xylem tissue of vascular plants. We begin this section with a brief discussion of the biophysical implications before concluding with a comment on the fluid physics.

Xylem cells carry water from roots to leaves in vascular plants. The conduits are connected by microscopic pit pores that permit water flow between adjacent channels (Fig. 1). Our experiments revealed strongly nonlinear flow properties of plant xylem that appear to be intricately linked to microscopic deformations in the cell-to-cell conduits. The artificial pit pore model supports this postulate. It is well established that a sufficiently large cell-to-cell pressure difference $\Delta p$ will cause the pores to close. This typically occurs to prevent gas pockets from migrating between cells $[7,8,12,23-25]$. At the interface between two liquid-filled neighbors, however, the pressure difference over the pit is widely believed to be too small to cause aspiration (i.e., $\Delta p / \sigma \ll 1$ ) [25-28]. However, the observed nonlinearity might also be an intrinsic functionality, enabling rapid flow control to prevent catastrophic water loss in response to strong localized transpiration stream fluctuations or tissue damage, where $\Delta p / \sigma \approx 1$, or to ensure a uniform supply of water throughout the organism [29,30]. This mode of flow control may also be crucial in allowing time for stomatal response in a highly variable environment of tree canopies (changing wind speed, variable vapor pressure deficit, exposure to sun flexes) where the dynamics of flux exceeds the response time of stomata. This would allow for a temporal limitation of flow, assuring that such short events do not cause long-lasting damage to xylem functionality. Fluid-structure interactions at the cell-to-cell level may thus be central to understanding the remarkable ability of plants to direct fluid flows without a centralized control unit.

The biological experiments provide inspiration for rethinking the fluid physics of man-made liquid handling systems. By exploiting the flow control principles deployed by plants, we have designed a variety of synthetic flow controllers. These rely on operating at or near the critical point $\Delta p / \sigma \approx 1$ where individual valves alternate between the open or closed state. The applications considered include a constant current source, a reverse Ohm controller, and a fluidic gate. The 
systems operate autonomously with no energy input or sensor feedback. Notably, we have shown that the framework is programmable, capable of encoding essentially any pressure-drop versus flowrate characteristic in a few simple components. This represents a potential future advance in flow control technology, complementary to existing devices [31-33].

\section{ACKNOWLEDGMENTS}

The authors wish to thank E. Hansen and S. R. Nimb for assistance in the construction of the experimental setup. This work was supported by research grants from VILLUM FONDEN (Grants No. 13166 and No. 37475) and the Independent Research Fund Denmark (Grant No. 9064-00069B).

Some of the authors have submitted patent applications related to the presented work.

[1] B. Mosadegh, P. Polygerinos, C. Keplinger, S. Wennstedt, R. F. Shepherd, U. Gupta, J. Shim, K. Bertoldi, C. J. Walsh, and G. M. Whitesides, Pneumatic networks for soft robotics that actuate rapidly, Adv. Funct. Mater. 24, 2163 (2014).

[2] S. Ghosh, K. Aggarwal, T. U. Vinitha, T. Nguyen, J. Han, and C. H. Ahn, A new microchannel capillary flow assay (MCFA) platform with lyophilized chemiluminescence reagents for a smartphone-based POCT detecting malaria, Microsyst. Nanoeng. 6, 5 (2020).

[3] R. Prantil-Baun, R. Novak, D. Das, M. R. Somayaji, A. Przekwas, and D. E. Ingber, Physiologically based pharmacokinetic and pharmacodynamic analysis enabled by microfluidically linked organs-on-chips, Annu. Rev. Pharmacol. Toxicol. 58, 37 (2018).

[4] A. Sontheimer-Phelps, B. A. Hassell, and D. E. Ingber, Modelling cancer in microfluidic human organson-chips, Nat. Rev. Cancer 19, 65 (2019).

[5] R. W. Coates, M. J. Delwiche, A. Broad, and M. Holler, Wireless sensor network with irrigation valve control, Comput. Electron. Agricult. 96, 13 (2013).

[6] O. Adeyemi, I. Grove, S. Peets, and T. Norton, Advanced monitoring and management systems for improving sustainability in precision irrigation, Sustainability 9, 353 (2017).

[7] B. Choat, S. Jansen, T. J. Brodribb, H. Cochard, S. Delzon, R. Bhaskar, S. J. Bucci, T. S. Feild, S. M. Gleason, U. G. Hacke et al., Global convergence in the vulnerability of forests to drought, Nature (London) 491, 752 (2012).

[8] B. Choat, T. J. Brodribb, C. R. Brodersen, R. A. Duursma, R. López, and B. E. Medlyn, Triggers of tree mortality under drought, Nature (London) 558, 531 (2018).

[9] J. Bauch, W. Liese, and R. Schultze, The morphological variability of the bordered pit membranes in gymnosperms, Wood Sci. Technol. 6, 165 (1972).

[10] See Supplemental Material at http://link.aps.org/supplemental/10.1103/PhysRevFluids.6.123102 for additional details on materials and methods.

[11] N. M. Holbrook and M. A. Zwieniecki, Vascular Transport in Plants (Elsevier, New York, 2011).

[12] K. H. Jensen, K. Berg-Sørensen, H. Bruus, N. M. Holbrook, J. Liesche, A. Schulz, M. A. Zwieniecki, and T. Bohr, Sap flow and sugar transport in plants, Rev. Mod. Phys. 88, 035007 (2016).

[13] N. G. McDowell and C. D. Allen, Darcy's law predicts widespread forest mortality under climate warming, Nat. Clim. Change 5, 669 (2015).

[14] G. Comstock and W. Côté, Factors affecting permeability and pit aspiration in coniferous sapwood, Wood Sci. Technol. 2, 279 (1968).

[15] K. Park, A. Tixier, A. Christensen, S. Arnbjerg-Nielsen, M. Zwieniecki, and K. Jensen, Viscous flow in a soft valve, J. Fluid Mech. 836, R3-1 (2018).

[16] M. Capron, P. Tordjeman, F. Charru, E. Badel, and H. Cochard, Gas flow in plant microfluidic networks controlled by capillary valves, Phys. Rev. E 89, 033019 (2014).

[17] F. Johansen, Flow through pipe orifices at low Reynolds numbers, Proc. R. Soc. London A 126, 231 (1930). 
[18] M. H. Zimmermann, Xylem Structure and the Ascent of Sap (Springer Science \& Business Media, New York, 2013).

[19] E. Ventsel and T. Krauthammer, Thin Plates and Shells (Marcel Dekker, New York, 2001).

[20] N. Rubido, C. Grebogi, and M. S. Baptista, General analytical solutions for dc/ac circuit-network analysis, Eur. Phys. J.: Spec. Top. 226, 1829 (2017).

[21] H. A. Core, Wood Structure and Identification (Syracuse University Press, Syracuse, NY, 1979).

[22] I. Daubechies, A. Grossmann, and Y. Meyer, Painless nonorthogonal expansions, J. Math. Phys. 27, 1271 (1986).

[23] F. Lens, A. Tixier, H. Cochard, J. S. Sperry, S. Jansen, and S. Herbette, Embolism resistance as a key mechanism to understand adaptive plant strategies, Curr. Opin. Plant Biol. 16, 287 (2013).

[24] P. J. Schulte and U. G. Hacke, Solid mechanics of the torus-margo in conifer intertracheid bordered pits, New Phytol. 229, 1431 (2021).

[25] B. Beikircher, T. Ameglio, H. Cochard, and S. Mayr, Limitation of the cavitron technique by conifer pit aspiration, J. Expt. Bot. 61, 3385 (2010).

[26] S. Gregory and J. Petty, Value action of bordered pits in conifers, J. Expt. Bot. 24, 763 (1973).

[27] D. Chapman, R. Rand, and J. Cooks, A hydrodynamical model of bordered pits in conifer tracheids, J. Theor. Biol. 67, 11 (1977).

[28] T. Hölttä, T. Vesala, and E. Nikinmaa, A model of bubble growth leading to xylem conduit embolism, J. Theor. Biol. 249, 111 (2007).

[29] M. B. Kirkham, Principles of Soil and Plant Water Relations (Academic Press, New York, 2014).

[30] E. E. Farmer, Leaf Defence (Oxford University Press, Oxford, 2014).

[31] P. Shamshery, R.-Q. Wang, D. V. Tran, and A. G. Winter V, Modeling the future of irrigation: A parametric description of pressure compensating drip irrigation emitter performance, PLoS ONE 12, e0175241 (2017).

[32] E. P. Kartalov, C. Walker, C. R. Taylor, W. F. Anderson, and A. Scherer, Microfluidic vias enable nested bioarrays and autoregulatory devices in Newtonian fluids, Proc. Natl. Acad. Sci. USA 103, 12280 (2006).

[33] H. Xia, J. Wu, and Z. Wang, The negative-differential-resistance (NDR) mechanism of a hydroelastic microfluidic oscillator, J. Micromech. Microeng. 27, 075001 (2017). 